\title{
Optimal Peer-to-Peer Technique for Massive Content Distribution
}

\author{
Xiaoying Zheng, Chunglae Cho and Ye Xia \\ Computer and Information Science and Engineering Department \\ University of Florida \\ Email: $\{$ xiazheng, ccho, yx1\}@cise.ufl.edu
}

\begin{abstract}
A distinct trend has emerged that the Internet is used to transport data on a more and more massive scale. Capacity shortage in the backbone networks has become a genuine possibility, which will be more serious with fiber-based access. The problem addressed in this paper is how to conduct massive content distribution efficiently in the future network environment where the capacity limitation can equally be at the core or the edge. We propose a novel peer-to-peer technique as a main content transport mechanism to achieve efficient network resource utilization. The technique uses multiple trees for distributing different file pieces, which at the heart is a version of swarming. In this paper, we formulate an optimization problem for determining an optimal set of distribution trees as well as the rate of distribution on each tree under bandwidth limitation at arbitrary places in the network. The optimal solution can be found by a distributed algorithm. The results of the paper not only provide stand-alone solutions to the massive content distribution problem, but should also help the understanding of existing distribution techniques such as BitTorrent or FastReplica.
\end{abstract}

Index Terms-Content Distribution, Peer-to-peer Networks, Multicast, Optimization, Bandwidth Allocation, Congestion Control

\section{INTRODUCTION}

One of the distinct trends is that the Internet is being used to transfer content on a more and more massive scale. The grass root initiative for massive content distribution by the user and research community has been a peer-to-peer $(\mathrm{P} 2 \mathrm{P})$ networking technique known as swarming. In a swarming session, the file to be distributed is broken into many chunks at the original source, which are then spread out across the peers in a fashion that the sets of chunks at different peers are substantially different. Subsequently, the peers can exchange the chunks with each other to speed up the distribution process. The above description of swarming does not specify the precise manner at which the chunks are initially spread out or the manner at which the peers exchange them later. In fact, many different ways of swarming have been proposed, such as BitTorrent [1], FastReplica [2], [3], Bullet [4], [5], Chunkcast [6], CoBlitz [7], and Julia [8]. The most popular one among them is the BitTorrent protocol.

Swarming enables content providers with poor capacity to reach a large number of audience, allows rapid deployment of a large distribution system with minimum infrastructure support, and is increasingly used in mainstream and critical network services. On the negative side, swarming traffic has made capacity shortage in the backbone networks a genuine possibility, which will become more serious with fiber-based access ${ }^{1}$. As an example, with its early adoption of FTTH, by 2005 , Japan already saw $62 \%$ of its backbone network traffic being from residential users to users, which was consumed by content downloading or $\mathrm{P} 2 \mathrm{P}$ file sharing; the fiber users were responsible for $86 \%$ of the inbound traffic; and the traffic was rapidly increasing, by $45 \%$ that year [11].

The problem addressed in this paper is how to conduct massive content distribution efficiently in the future network environment where the capacity limitation can equally be at the core or the edge. The proposed solution is a class of improved swarming techniques, known as optimal swarming. In this paper, swarming is viewed not just as a casual technique for end-user file-sharing applications. The benefits of swarming rest upon the fact it is an advanced form of networking mechanism, more advanced and more powerful than all previous distribution schemes, including IP or application-level multicast (e.g. [12]), network cache systems and existing content distribution networks (e.g., Akamai [13]). As will be seen, swarming can be thought as distributing content on multiple multicast trees. When done properly, it provides the most efficient utilization of the network capacity, a remedy for backbone congestion, or gives the fastest distribution.

For illustration of the main ideas in this paper, consider the toy example in Fig. 1. The numbers associated with the links are their capacities. Suppose a large file is split into many chunks at source node 1 . We wish to find the fastest way to distribute all chunks to receivers 2 and $3 .{ }^{2}$ We impose no restriction on how peers can help each other in the distribution process. Let us focus on a fixed chunk and consider how it can be distributed to the receivers. With some thoughts, it can be argued that, when the delay is not modeled, the path should be a tree rooted at the source and covering both receivers. All possible distribution trees are shown in Fig. 2. The question becomes how to assign the chunks to different distribution trees so that the distribution time is minimized, subject to the link capacity constraint. For this simple example, it is easy to

\footnotetext{
${ }^{1}$ At the present, telecom companies are aggressively rolling out fiber-to-the home $($ FTTH) or its variants. Verizon is building a nationwide FTTH network in the US, to be completed by 2010. Japan had 5.6 million FTTH subscribers by June, 2006, is on an exponential upward ramp to have 30 million by 2010 [9]. The speed of the access fiber is currently at $100 \mathrm{Mbps}$ or lower, heading to $1 \mathrm{Gbps}$ by 2020 and is likely to reach $10 \mathrm{Gbps}$ thereafter [10].

${ }^{2}$ As we will show, the objective of minimizing the distribution time is equivalent to maximizing the distribution throughput, which is also equivalent to minimizing the network congestion.
} 
see that distributing the chunks in 1:2 ratio on the second and third tree, while leaving the first unused, is optimal.

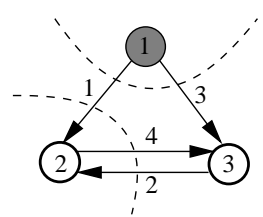

Fig. 1. Node 1 sends the file to node 2 and 3.
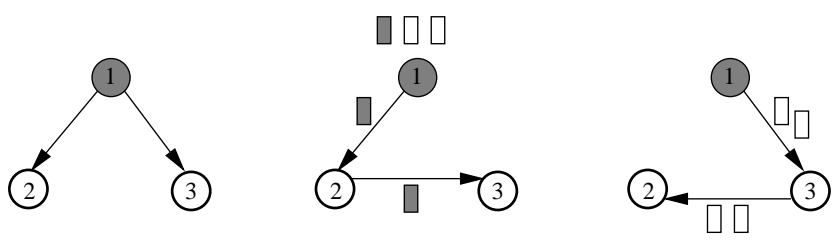

Fig. 2. All possible distribution trees for the example in Fig. 1.

The ideas contained in the toy example are also given in [14]. That work focuses on how to compute the maximum throughput, which leads to the fastest distribution. Our contribution in this paper is on developing distributed algorithms to identify and use the optimal distribution trees, and at the same time, allocate correct bandwidth on the selected trees.

Our approach illustrated by the toy example can be contrasted with existing P2P distribution systems or techniques [1]-[8]. Being originally designed for end-system file-sharing applications, the current swarming systems leave much room for improvement. Most systems only select and solve part of the problem and do so with poorly understood heuristics. Not enough is known on how well they work or how much improvement remains possible. They have user-centric performance objectives, but mostly ignore the important networkcentric objectives, such as minimizing network congestion. The bandwidth bottleneck is often assumed at the access links, rather than throughout the network. Our solution will be able to automatically adapt to capacity constraint anywhere in the network. Furthermore, within our framework, we are able to solve four separate problems jointly and optimally: peer selection (from which peer to download), chunk selection (which chunks to download from a peer), distribution tree selection and bandwidth allocation. In contrast, other P2P systems solve one or two of these problems in isolation, typically using ad-hoc approaches.

The paper is organized as follows. The models and problem formulations are given in Section II. The distributed algorithm is given in Section III. In Section IV, we discuss practical issues in applying our algorithm to realistic settings, such as scalability and coping with network dynamics and churn. In Section V, we evaluate the performance of our algorithm, including a comparison with BitTorrent and FastReplica. In Section VI, we discuss additional related work. The conclusion is drawn in Section VII.

\section{Problem Description}

We will start with a formulation for optimal content distribution on a generic network. It turns out the problem is difficult on an arbitrary network. However, for overlay content distribution, the problem is far easier. We will give formulations for two possible scenarios of overlay distribution.

\section{A. Optimal Multicast Tree Packing}

Let the network be represented by $G=(V, E)$, where $V$ is the set of nodes and $E$ is the set of links. The capacity associated with each link $e \in E$ is $c_{e}$. The utilization of link $e$, a measure of link congestion, is denoted by $\mu_{e}$. We define a multicast session as a group of nodes (members) exchanging the same file. In a session, some members own some distinct chunks of a large file (The case of overlapping content at different nodes requires a minor extension, which will be discussed in Section IV-A.) and we call those members sources of the session. A reasonable assumption about a session is that all members in the session are interested in the file, and at the end of file distribution, every member in the session will have a complete copy of the file.

Let $M$ be the set of all multicast sessions. For each session $m \in M$, let $V^{(m)} \subseteq V$ represent the set of members in session $m$, and let $S^{(m)} \subseteq V^{(m)}$ be the set of sources in session $m$. For each source $s \in S^{(m)}$, let $L_{s}^{(m)}$ be the total size of the file chunks at source $s$ for session $m$. Let the set of all possible multicast trees spanning all members in the session rooted at source $s \in S^{(m)}$ be denoted by $T_{s}^{(m)}$. A multicast tree may contain nodes not in the session, in which case the tree is called a Steiner tree. In the case where all nodes on the tree belong to the session, the multicast tree is called a spanning tree, meaning it spans the multicast session (rather than the whole network $V$ ). For the $i^{t h}$ tree $t_{s, i}^{(m)} \in T_{s}^{(m)}$, where the order of indexing is arbitrary, denote $z_{s, i}^{(m)}$ to be the sending rate on tree $t_{s, i}^{(m)}$ from the root $s$.

A straightforward objective is to minimize the overall downloading time for all multicast sessions, which is to minimize the worst downloading time associated with any source $s$ in any session $m$. With some thought, we see that no difference is made in terms of the achievable downloading time if we assume that all sources in all sessions finish their distribution at the same time. We can then minimize this common duration $t$. Let the total rate on a link $e \in E$ be denoted by $x_{e}$. It is equal to the sum of all the rates on all the trees passing through link $e$, across all sessions and all sources. That is,

$$
x_{e}=\sum_{m \in M} \sum_{s \in S^{(m)}} \sum_{i: e \in t_{s, i}^{(m)}} z_{s, i}^{(m)} .
$$

The optimization problem is as follows.

$$
\begin{array}{cl}
\min & t \\
\text { s.t. } & t \sum_{i=1}^{\left|T_{s}^{(m)}\right|} z_{s, i}^{(m)}=L_{s}^{(m)}, \quad \forall s \in S^{(m)}, \forall m \in M \\
& x_{e} \leq c_{e}, \quad \forall e \in E \\
z_{s, i}^{(m)} \geq 0, \quad \forall i=1, \cdots,\left|T_{s}^{(m)}\right|, \forall s \in S^{(m)}, \forall m \in M .
\end{array}
$$


Condition (3) says that, if one looks at all the multicast trees rooted at a source $s$ for a session $m$, the sum of the distribution rates on all these trees, multiplied by the distribution time, should be equal to the total size of all the file chunks stored at source $s$ for session $m$. This means that every bit of the file stored at $s$ must be transmitted exactly once. A moment of thinking reveals that nothing is gained by transmitting the same bit more than once. Condition (4) is the link capacity constraint. At each link $e$, the flow rate on the link should be no greater than the link capacity, $c_{e}$.

It turns out the above problem is equivalent to a minimizingcongestion problem. This is immediate if we define $y_{s, i}^{(m)}=$ $t z_{s, i}^{(m)}$ and make the substitution of variables. But, we will do this a little differently for ease of interpretation. Let $z_{s}^{(m)}=\sum_{i=1}^{\left|T_{s}^{(m)}\right|} z_{s, i}^{(m)}$ be the total sending rate at a source node $s$ of session $m$. Select a set of constants $\left\{r_{s}^{(m)}\right\}$, each being proportional to $L_{s}^{(m)}$ with the same constant proportional factor. Each $r_{s}^{(m)}$ is understood as a rate. Consider a feasible solution $\left\{z_{s, i}^{(m)}\right\}$ and $t$. By (3), $z_{s}^{(m)}$ is proportional to $L_{s}^{(m)}$, the total size of the chunks at $s$ for session $m$. Then, $z_{s}^{(m)}=\gamma r_{s}^{(m)}$, for some constant $\gamma>0$. Next, define $\mu=1 / \gamma$. We then make the substitution of variables by $t=\frac{\mu L_{s}^{(m)}}{r_{s}^{(m)}}$, and redefine $z_{s, i}^{(m)}$ to be $\mu z_{s, i}^{(m)}$. We get the following minimizingcongestion formulation.

$$
\begin{array}{ll}
\min & \mu \\
\text { s.t. } & \sum_{i=1}^{\left|T_{s}^{(m)}\right|} z_{s, i}^{(m)}=r_{s}^{(m)}, \quad \forall s \in S^{(m)}, \forall m \in M \\
& x_{e} \leq \mu c_{e}, \quad \forall e \in E \\
z_{s, i}^{(m)} \geq 0, \quad \forall i=1, \cdots,\left|T_{s}^{(m)}\right|, \forall s \in S^{(m)}, \forall m \in M .
\end{array}
$$

In the above formulation, $r_{s}^{(m)}$ can be understood as the demanded rate, and $\mu$ is the maximum link utilization, which also measures the worst link congestion. The problem is to minimize the worst link congestion subject to the fulfillment of all demanded rates.

Thus, we have two equivalent views of optimal multicast tree packing. In the first view, the objective is to minimize the overall distribution time (or maximize the distribution throughput) while satisfying the link capacity constraint. In the second view, the objective is to best balance the network load while satisfying the rate demand for all sources and all sessions.

Another minor reformulation will be helpful later. Let $\mu_{e}$ stand for the utilization of link $e$, and let $\vec{\mu}$ denote the vector of $\mu_{e}$ over all links. Let $\|\vec{\mu}\|_{\infty}$ denote the maximum norm, i.e., $\|\vec{\mu}\|_{\infty}=\max _{e \in E} \mu_{e}$. The above minimizing-congestion formulation is equivalent to the following.

$$
\begin{array}{ll}
\min & || \vec{\mu} \|_{\infty} \\
\text { s.t. } & \sum_{i=1}^{\left|T_{s}^{(m)}\right|} z_{s, i}^{(m)}=r_{s}^{(m)}, \quad \forall s \in S^{(m)}, \forall m \in M \\
& x_{e}=\mu_{e} c_{e}, \quad \forall e \in E \\
z_{s, i}^{(m)} \geq 0, \quad \forall i=1, \cdots,\left|T_{s}^{(m)}\right|, \forall s \in S^{(m)}, \forall m \in M .
\end{array}
$$

The optimization problem proposed so far is equivalent to the problem of packing Steiner trees [15], [16], which is computationally intractable. Fortunately, for P2P content distribution, the problem becomes simpler. The main reason is that the overlay network of each session consists of exactly those nodes in the session. Given such an overlay network, any Steiner tree that covers all nodes of the session is in fact a spanning tree. We will show later that the algorithm used to solve the optimization problem involves a minimum-cost spanning tree problem in each iteration. Should some Steiner node exist, it would have involved a minimum-cost Steiner tree problem. The former is far more tractable than the latter NP-hard problem. One should be reminded that, although the computation for the overlay network case is far easier, the achievable performance is sub-optimal since the overlay edges are determined by the fixed underlay routing.

Unlike the inflexible underlay routing, the bandwidth of the overlay edge may have alternatives. Next, we consider two cases, both of which can be useful.

\section{B. Fixed Overlay Link Bandwidth}

In this case, the bandwidth of each overlay link is a fixed constant ${ }^{3}$ determined by a bandwidth allocation scheme external to our problem. For instance, the bandwidth may be determined by the end-to-end TCP control, or by bandwidth allocation algorithms that enforce other allocation policies such as the max-min fairness [17]. We assume the overlay nodes know about the bandwidth on each overlay link. For instance, in the case of TCP, the overlay link bandwidth can be measured. When the overlay link bandwidth is fixed, different distribution sessions become decoupled. We then have $|M|$ totally independent overlay networks. The original optimization problem becomes separated to $|M|$ identical but independent optimization problems. The solution to this problem will require running algorithms only at the overlay nodes, making deployment easy.

We illustrate this by focusing on one of the overlay networks, which corresponds to one session. Let $\hat{G}=(\hat{V}, \hat{E})$ represent the overlay network. For all other notations, since there is no danger of confusing them with earlier definitions, we will re-define them. The bandwidth associated with each overlay link $e \in \hat{E}$ is $c_{e}$, which is allocated already and is a constant. The utilization of overlay link $e$ is denoted by $\mu_{e}$. Assume $S \subseteq \hat{V}$ is the set of sources. Let $T_{s}$ represent the set of all possible (overlay) multicast trees rooted at source $s$ spanning all overlay nodes. Let $t_{s, i} \in T_{s}$ be the $i^{t h}$ (overlay) multicast tree and $z_{s, i}$ be the associated sending rate on tree $t_{s, i}$. The rate on the overlay link $e \in \hat{E}$ is

$$
x_{e}=\sum_{s \in S} \sum_{i: e \in t_{s, i}} z_{s, i}
$$

\footnotetext{
${ }^{3}$ By fixed overlay bandwidth, we do not mean the bandwidth may not vary over time. We simply mean that the overlay link bandwidth is not determined by our algorithm and is known at the time of running the algorithm.
} 
The optimization problem is now

$$
\begin{aligned}
& \min \|\vec{\mu}\|_{\infty} \\
& \text { s.t. } \sum_{i=1}^{\left|T_{s}\right|} z_{s, i}=r_{s}, \quad \forall s \in S \\
& x_{e}=\mu_{e} c_{e}, \quad \forall e \in \hat{E} \\
& z_{s, i} \geq 0, \quad \forall i=1, \cdots,\left|T_{s}\right|, \quad \forall s \in S .
\end{aligned}
$$

\section{Optimally Allocated Overlay Bandwidth}

In this subsection, we consider an alternative scenario with better performance. Instead of relying on TCP to allocate the overlay bandwidth, leading to the partition of the overall network into multiple independent overlay networks, we will incorporate overlay bandwidth allocation into the optimization problem. Note that different sessions are coupled together by the sharing of the underlay links. The solution to this problem will require cooperation from the physical links. But, it is possible to modify the problem slightly and run the algorithm at only bottleneck links, such as the inter-ISP slow links.

Let $\hat{G}^{(m)}=\left(\hat{V}^{(m)}, \hat{E}^{(m)}\right)$ represent the overlay network for each session $m$. For each overlay link $\hat{e} \in \hat{E}^{(m)}$, the notation $e \in \hat{e}$ for some underlay link $e \in E$ means that link $e$ is on overlay link $\hat{e}$, which is itself an underlay path. For each session $m$, let $T_{s}^{(m)}$ be the set of all possible spanning trees on $\hat{G}^{(m)}$ rooted at source $s$, and $t_{s, i}^{(m)}$ be the $i^{t h}$ tree in $T_{s}^{(m)}$. Then, the total rate on a physical link $e \in E, x_{e}$, is given by

$$
x_{e}=\sum_{m \in M} \sum_{s \in S^{(m)}} \sum_{\hat{e} \in \hat{E}^{(m)}: e \in \hat{e}} \sum_{i: \hat{e} \in t_{s, i}^{(m)}} z_{s, i}^{(m)} .
$$

The optimization problem is exactly written as in (10)-(13).

\section{Distributed Algorithm: Diagonally ScAled GRADIENT PROJECTION}

Note that $\min \|\vec{\mu}\|_{\infty}$ has the same solution as $\min \| \vec{\mu}+$ $\vec{\kappa} \|_{\infty}$, where $\vec{\kappa}=(\kappa, \ldots, \kappa)$ for some small constant $\kappa \geq 0$. More precisely, we replace the objective function $\|\vec{\mu}\|_{\infty}$ by $\|\vec{\mu}+\vec{\kappa}\|_{\infty}$ in (10) and keep the same constraints. $\vec{\kappa}$ serves as a regularization term. The strictly positive vector $\vec{\kappa}$ (i.e., $\kappa>0$ ) guarantees a global geometric convergence rate of our gradient projection algorithm; if $\vec{\kappa}=\overrightarrow{0}$, we can only claim that our gradient projection algorithm converges to one optimal solution globally.

\section{A. Fixed Overlay Link Bandwidth}

The goal of minimizing $\|\vec{\mu}+\vec{\kappa}\|_{\infty}$ is to balance the network load. The same objective can be achieved by minimizing $\sum_{e \in \hat{E}} \hat{f}_{e}\left(x_{e}\right)$, where $\hat{f}_{e}$ is some convex increasing function on $x_{e} \geq 0$. Such an objective function discourages large link rate. One such function is the $q$ norm $\|\vec{\mu}+\vec{\kappa}\|_{q}=$ $\left(\sum_{e \in \hat{E}}\left(\mu_{e}+\kappa\right)^{q}\right)^{1 / q}$. In fact, $\|\vec{\mu}+\vec{\kappa}\|_{\infty}$ can be approximated by $\|\vec{\mu}+\vec{\kappa}\|_{q}$ : as $q \rightarrow \infty,\|\vec{\mu}+\vec{\kappa}\|_{q} \rightarrow\|\vec{\mu}+\vec{\kappa}\|_{\infty}$. We will assume $q \geq 2$ throughout. Since $\min \|\vec{\mu}+\vec{\kappa}\|_{q}$ is equivalent to $\min \|\vec{\mu}+\vec{\kappa}\|_{q}^{q}$, after substitution of $\mu_{e}$ with $\frac{x_{e}}{c_{e}}$, (15) becomes

$$
\begin{aligned}
& \min \sum_{e \in \hat{E}}\left(\frac{x_{e}}{c_{e}}+\kappa\right)^{q} \\
& \text { s.t. } \sum_{i=1}^{\left|T_{s}\right|} z_{s, i}=r_{s}, \quad \forall s \in S \\
& z_{s, i} \geq 0, \quad \forall i=1, \cdots,\left|T_{s}\right|, \quad \forall s \in S .
\end{aligned}
$$

For optimization problems with the simplex constraint of the form (18), the optimality condition is especially simple [18]. It has been shown in [19] and [20] that there exists a special gradient projection algorithm. For our case, the gradient projection algorithm can also be easily extended to an equally simple scaled version. The latter overcomes the issue that our problem may be ill-conditioned, and hence, drastically improves the algorithm's convergence time. Our computational experiences have shown that the scaled gradient algorithm is much faster than the unscaled algorithm or the subgradient algorithm. The latter is often used in network optimization problems.

Another difficulty is the large number of possible spanning trees, and hence, the large number of variables. Fortunately, the algorithm does not maintain all possible spanning trees. The following steps take place for every source at the overlay network level. The algorithm starts out with one or few spanning trees. In each iteration, a cost is assigned to each (overlay) link to reflect the current link congestion. Then, a minimumcost spanning tree can be computed. The algorithm shifts an appropriate amount of traffic (rate) from each currently maintained spanning tree to the minimum-cost tree. The new minimum-cost tree enters the current collection of spanning trees. Some previous spanning tree may leave the collection if its distribution rate is reduced to zero.

We next illustrate some details. Let $T=\bigcup_{s \in S} T_{s}$ be the collection of all multicast trees rooted at any source. Let $z$ be the vector $\left(z_{s, i}\right)$ where $s \in S, i=1, \ldots,\left|T_{s}\right|$, with an arbitrary indexing order for the sources. In problem (17), let the feasible set defined by (18) and (19) be denoted by $\mathcal{Z}$.

For each overlay link $e \in \hat{E}$, recall that $x_{e}$ is the aggregate flow rate it carries. Let $x$ be the vector $\left(x_{e}\right)_{e \in \hat{E}}$. Let $H$ denote the $|\hat{E}| \times|T|$ link-tree incidence matrix associated with the trees in $T$ (i.e. $[H]_{e t}=1$ if link $e$ lies on tree $t$; and $[H]_{e t}=0$ otherwise). Obviously, $x=H z$. Now define $\hat{f}_{e}\left(x_{e}\right)=\left(x_{e} / c_{e}+\kappa\right)^{q}$ and $\hat{f}(x)=\sum_{e \in \hat{E}} \hat{f}_{e}\left(x_{e}\right)$. The objective function, denoted by $f(z)$, is given by

$$
f(z)=\hat{f}(H z)=\sum_{e \in \hat{E}} \hat{f}_{e}\left(x_{e}\right)=\sum_{e \in \hat{E}}\left(\frac{x_{e}}{c_{e}}+\kappa\right)^{q},
$$

and (17) can be written as

$$
\begin{array}{cc}
\min & f(z)=\hat{f}(H z) \\
\text { s.t. } & z \in \mathcal{Z} .
\end{array}
$$

The derivative of the objective function $f(z)$ with respect to $z_{s, i}$ is given by

$$
\frac{\partial f(z)}{\partial z_{s, i}}=\sum_{e \in t_{s, i}} \frac{\partial \hat{f}\left(x_{e}\right)}{\partial x_{e}}=\sum_{e \in t_{s, i}} \frac{q}{c_{e}}\left(\frac{x_{e}}{c_{e}}+\kappa\right)^{q-1} .
$$


Note that $\frac{\partial \hat{f}\left(x_{e}\right)}{\partial x_{e}}$ is the so-called first-derivative link cost of link $e$ [18], [20]. It reflects the current congestion level at link $e . \frac{\partial f(z)}{\partial z_{s, i}}$ is the first-derivative cost of the tree $t_{s, i}$, which is equal to the sum of the first-derivative costs of the links on the tree. It reflects the current congestion level of the tree $t_{s, i}$. The first-derivative tree cost is an important quantity. We will see later that our algorithm is to shift flows from trees with higher costs to the minimum-cost tree. The second derivative of $f(z)$ will be used in the scaling of the algorithm. With respect to $z_{s, i}$ and $z_{s, j}$, it is given by

$$
\frac{\partial^{2} f(z)}{\partial z_{s, i} \partial z_{s, j}}=\sum_{e \in t_{s, i} \cap t_{s, j}} \frac{q(q-1)}{c_{e}^{2}}\left(\frac{x_{e}}{c_{e}}+\kappa\right)^{q-2} .
$$

For each $s \in S$, let $i_{s}$ be the index of a minimum-cost tree rooted at $s$ (i.e., with $s$ as the source). That is,

$$
i_{s}(z)=\operatorname{argmin}_{\left\{i: t_{s, i} \in T_{s}\right\}}\left\{\frac{\partial f(z)}{\partial z_{s, i}}\right\} .
$$

If there are multiple minimum-cost trees, we choose an arbitrary one. Since the feasible set $\mathcal{Z}$ is a convex set and the objective function is a convex function, we can characterize an optimal solution $z^{*}$ to the problem (17) by the following optimality condition.

$$
\sum_{s \in S} \sum_{i: t_{s, i} \in T_{s}} \frac{\partial f\left(z^{*}\right)}{\partial z_{s, i}}\left(z_{s, i}-z_{s, i}^{*}\right) \geq 0, \forall z \in \mathcal{Z} .
$$

This optimality condition can be equivalently written as, for any source $s \in S$,

$$
z_{s, i}^{*}>0 \text { only if }\left[\frac{\partial f\left(z^{*}\right)}{\partial z_{s, j}} \geq \frac{\partial f\left(z^{*}\right)}{\partial z_{s, i}}, \forall t_{s, j} \in T_{s}\right] .
$$

That is, for every source, only those trees with the minimum first-derivative cost carry positive amount of flow. This intuitively suggests that, in the algorithm, we should shift flow to the minimum-cost trees from other trees.

It turns out this is exactly what the gradient projection algorithm does. We will develop the gradient projection algorithm following the proposal in [20] to solve the problem (17). But we will add diagonal scaling to speed up the algorithm's convergence time.

The equality constraint in (18) implies that, for each source, one of the variables depends completely on the rest of the variables. We can eliminate this variable and have a problem with fewer variables. To be concrete, at a feasible vector $z$, let's eliminate the variable $z_{s, i_{s}}$ for each $s \in S$. Define a new objective function $g(\hat{z})$ on $\mathbb{R}^{|T|-|S|}$, where $\hat{z}$ consists of the remaining $z_{s, i}$ 's after $z_{s, i_{s}}$ is eliminated for each $s$. Without loss of generality, suppose, for each source $s, i_{s}$ corresponds to the tree with the largest index, i.e., $i_{s}=\left|T_{s}\right|$. Also suppose the sources are index from 1 to $|S|$. Then,

$$
\begin{aligned}
\hat{z}= & \left(z_{1,1}, \ldots, z_{1,\left|T_{1}\right|-1} ; z_{2,1}, \ldots, z_{2,\left|T_{2}\right|-1}\right. \\
& \left.\ldots ; z_{|S|, 1}, \ldots, z_{|S|, \mid T_{|S|}}\right) .
\end{aligned}
$$

We will call the domain of $g$ where $\hat{z}$ lies the reduced domain. We let

$$
\begin{gathered}
g(\hat{z})=f\left(z_{1,1}, \ldots, z_{1,\left|T_{1}\right|-1}, r_{1}-\sum_{j=1}^{\left|T_{1}\right|-1} z_{1, j} ;\right. \\
z_{2,1}, \ldots, z_{2,\left|T_{2}\right|-1}, r_{2}-\sum_{j=1}^{\left|T_{2}\right|-1} z_{2, j} ; \ldots ; \\
\left.z_{|S|, 1}, \ldots, z_{|S|,\left|T_{|S|}-1\right|}, r_{|S|}-\sum_{j=1}^{\left|T_{|S|}\right|-1} z_{|S|, j}\right) .
\end{gathered}
$$

The optimization problem in (17)- (19) is equivalent to

$$
\begin{aligned}
& \min g(\hat{z}) \\
& \text { s.t. } \hat{z} \geq 0 .
\end{aligned}
$$

This problem can be solved by the gradient projection algorithm.

$$
\hat{z}(k+1)=[\hat{z}(k)-\delta(k) \nabla g(\hat{z}(k))]_{+},
$$

where $\delta(k)$ is a positive step size and [ $]_{+}$is the projection operator on $\hat{z} \geq 0$. In this case, $[y]_{+}$just means that, if $y_{i}$ is a component of $y$, we take $\max \left(y_{i}, 0\right)$ as the corresponding component of the vector $[y]_{+}$. The key is to compute $\nabla g(\hat{z}(k))$. Let $i_{s}(k)$ be a short hand for $i_{s}(z(k))$. It is easy to show, for $s \in S$ and $i \neq i_{s}(k)$,

$$
\frac{\partial g(\hat{z}(k))}{\partial z_{s, i}}=\frac{\partial f(z(k))}{\partial z_{s, i}}-\frac{\partial f(z(k))}{\partial z_{s, i_{s}(k)}} .
$$

The first derivatives are given in (22).

The algorithm in (29) is actually the constrained steepestdescent algorithm. It is well-known that the steepest-descent algorithm can be slow if the optimization problem is illconditioned. It happens that the minimizing-congestion type of network problems is often ill-conditioned. In our case, the problem becomes more ill-conditioned when the parameter $q$ in the $q$ norm becomes larger. An ultimate solution to an ill-conditioned problem is Newton's algorithm. However, Newton's algorithm is generally very complex since it requires the inverse of the Hessian matrix of the objective function. For large problems, this computation is generally impractical. We will next develop the diagonally scaled gradient algorithm, which is a much simpler alternative and a good approximation of Newton's algorithm. The scaled gradient projection algorithm can be written as

$$
\hat{z}(k+1)=[\hat{z}(k)-\delta(k) D(k) \nabla g(\hat{z}(k))]_{+},
$$

where $D(k)$ is a positive definite matrix. For diagonal scaling, $D(k)$ is chosen to be a diagonal matrix.

$$
D(k)=\operatorname{diag}\left[\left(d_{s, i}(k)\right)^{-1}\right]_{s \in S, i \neq i_{s}(k)} .
$$

That is, the diagonal entry corresponding to $z_{s, i}(k)$ is chosen to be $\left(d_{s, i}(k)\right)^{-1}$. For each $s \in S$ and $i \neq i_{s}(k)$, the value of $d_{s, i}(k)$ is chosen to be

$$
d_{s, i}(k)=\frac{\partial^{2} g(\hat{z}(k))}{\partial z_{s, i}^{2}} .
$$


This way, the matrix $D(k)$ approximates the inverse of the Hessian of $g$ at $\hat{z}(k)$. For each $s \in S$ and $i \neq i_{s}(k)$, the second derivative of $g$ is further given by

$$
\frac{\partial^{2} g(\hat{z}(k))}{\partial z_{s, i}^{2}}=\frac{\partial^{2} f(z(k))}{\partial z_{s, i}^{2}}+\frac{\partial^{2} f(z(k))}{\partial z_{s, i_{s}(k)}^{2}}-2 \frac{\partial^{2} f(z(k))}{\partial z_{s, i} \partial z_{s, i_{s}(k)}} .
$$

The second derivatives are given in (23).

We can now collect different pieces of the development above and formally give the diagonally scaled gradient projection algorithm in the original domain where $z$ lies. A slight generalization is present in (35).

\section{Diagonally Scaled Gradient Projection Algorithm}

$$
\begin{gathered}
z(k+1)=\alpha(k) \bar{z}(k)+(1-\alpha(k)) z(k) \\
\bar{z}_{s, i}(k)= \\
\left\{\begin{array}{c}
{\left[z_{s, i}(k)-\delta(k) \cdot\left(d_{s, i}(k)\right)^{-1} \cdot\left(\frac{\partial f(z(k))}{\partial z_{s, i}}-\frac{\partial f(z(k))}{\left.\partial z_{s, i_{s}(k)}\right)}\right]_{+},\right.} \\
\text {if } i \neq i_{s}(k) ; \\
r_{s}-\sum_{1 \leq j \leq\left|T_{s}\right|, j \neq i_{s}(k)} \bar{z}_{s, j}(k), \quad \text { if } i=i_{s}(k),
\end{array}\right.
\end{gathered}
$$

with

$$
d_{s, i}(k)=\sum_{e \in t_{s, i} \cup t_{s, i_{s}(k)} \backslash t_{s, i} \cap t_{s, i_{s}(k)}} \frac{q(q-1)}{c_{e}^{2}}\left(\frac{x_{e}(k)}{c_{e}}+\kappa\right)^{q-2} .
$$

In (35), $\alpha(k)$ is a scalar on $[\underline{a}, 1]$, for some $\underline{a}, 0<\underline{a} \leq 1$. (35) says that the new rate vector at the $(i+1)^{\text {th }}$ iteration, $z(k+1)$, is on the line segment between $z(k)$ and $\bar{z}(k)$.

The main part of the algorithm is expression (36), which computes the end point of a feasible direction, $\bar{z}(k)$, entry by entry. There are three cases.

case 1 If a tree $t_{s, i}$ is not the chosen minimum-cost tree (with index $i_{s}(k)$ ) and $t_{s, i}$ has a positive flow, its rate will be reduced (more precisely, if $t_{s, i}$ is a minimumcost tree with positive flow rate but not the chosen minimum-cost tree, its rate will keep the same).

case 2 If a tree $t_{s, i}$ is not the chosen minimum-cost tree and the tree has zero flow rate, then the rate stays at 0 .

case 3 If $t_{s, i}$ is the chosen minimum-cost tree, the rate of the tree is increased so that the total rates of all trees rooted at $s$ will be equal to the demanded rate $r_{s}$.

Note that the description in case 3 ensures that $\bar{z}(k)$ is feasible (in $\mathcal{Z}$ ). Since $z(k)$ is also feasible, by (35), the new rate vector $z(k+1)$ is feasible. Hence, if we start with a feasible solution in $\mathcal{Z}, z(k)$ is in $\mathcal{Z}$ for all $k{ }^{4}$

What remains to be said is how much the rate is reduced in case 1. Note that the expression $\frac{\partial f(z(k))}{\partial z_{s, i}}-\frac{\partial f(z(k))}{\partial z_{s, i}(k)}$ is the difference in the first-derivative cost between the tree $t_{s, i}$ and the chosen minimum-cost tree, and the difference is always non-negative. Intuitively, the amount of reduction should be proportional to this difference. Indeed, if we ignore the factor $\left(d_{s, i}(k)\right)^{-1}$ in (36), the rate reduction is proportional

${ }^{4}(35)$ can be replaced with a more general update $z(k+1)=A(k) \bar{z}(k)+$ $(I-z(k)) z(k)$, where $A(k)$ is a $\sum_{s \in S}\left|T_{s}\right| \times \sum_{s \in S}\left|T_{s}\right|$ diagonal matrix with diagonal entries in the interval $[\underline{a}, 1]$, for some $\underline{a}, 0<\underline{a} \leq 1$. To ensure feasibility of $z(k+1)$ in $\mathcal{Z}$, it is required that $\sum_{1<i \leq\left|T_{s}\right|} a_{s, i}(k)\left(\bar{z}_{s, i}(k)-\right.$ $\left.z_{s, i}(k)\right)=0$, where $a_{s, i}(k)$ is a corresponding diagonal entry of $A(k)$. to the cost difference with the proportional constant (step size) $\delta_{s}(k)>0$.

The factor $\left(d_{s, i}(k)\right)^{-1}$ does the diagonal scaling, which can effectively deal with our ill-conditioned problem. The scaling factor $\left(d_{s, i}(k)\right)^{-1}$ can be understood as allowing different components of the vector $z$ to use different step sizes. Note that, in the expression for $d_{s, i}(k)$ in (37), which corresponds to the $i^{t h}$ tree, the sum is over the non-overlapping links between the $i^{t h}$ tree and the $i_{s}(k)^{t h}$ tree, the latter being the minimumcost tree.

The algorithm in (35)-(37) is a distributed one. In order to compute the tree cost, $\frac{\partial f(z)}{\partial z_{s, i}}$ in (22), and the scaling factor, $\left(d_{s, i}(k)\right)^{-1}$ in (37), each link $e$ can independently compute its corresponding term based on the local aggregate rate, $x_{e}$, passing through the link. Then, the tree cost and the scaling factor can be accumulated by the source $s$ based on the link values along the tree. To find the minimum-cost tree $i_{s}(k)$, each source needs to compute the minimum-cost directed spanning tree (MDSP). Both centralized and distributed algorithms exist for computing the MDSP [21] [22] [23]. Both achieve $O\left(n^{2}\right)$ time complexity for a complete graph with $n$ nodes. In the distributed version, the amount of information exchanged is also $O\left(n^{2}\right)$. In our implementation, each source collects the (overlay) link costs from all the receivers and uses a centralized algorithm to compute the MDSP. Other than that, the gradient algorithm is completely decentralized.

In addition to fast convergence, another strength of this gradient algorithm lies in that it avoids the enumeration of all possible spanning trees. The source only needs to manage the set of active multicast trees, i.e., those trees with positive flows. At each iteration, the source computes a new minimumcost tree. A non-active tree won't become active unless it is the minimum-cost tree. The source only adjusts the flow rates among the set of active trees. The set of active trees usually is not very large if the algorithm converges fast, since, at each iteration, at most one more tree becomes active. For the original linear model (15), there are at most $|\hat{E}|+|S|$ active trees in any extreme point solution. But since this gradient algorithm is a kind of interior point method, strictly speaking, $|\hat{E}|+|S|$ is not really an upper bound. Nevertheless, it should give a rough sense on what the bound might be.

We stress that the reason to apply the scaling factor is to counter the ill-conditioned problem when $q$ is large. In an ill-conditioned problem, single-unit changes of different variables have disproportionate effects on the cost (e.g., objective) change. For convergence, the step size in the iterative algorithm must be tuned according to the variables that cause large cost changes. However, such a step size can be too small for other variables, and as a result, they hardly change from iteration to iteration. The diagonally scaled algorithm essentially re-scales the variables so that single-unit changes in the scaled variables have similar effect on the cost objective. For our problem, the scaling has the simple interpretation that different trees use different step sizes, each roughly being proportional to a power of the worst link utilization on the tree. The resulting scaled algorithm is far superior to the plain gradient projection algorithm. 


\section{B. Optimally Allocated Overlay Bandwidth}

The problem described in Section II-C can be worked out in a similar way, leading to a scaled gradient projection algorithm. Substitute $\mu_{e}$ with $\frac{x_{e}}{c_{e}}$, the problem becomes

$$
\begin{array}{ll}
\min & \sum_{e \in E}\left(\frac{x_{e}}{c_{e}}+\kappa\right)^{q} \\
\text { s.t. } & \sum_{i=1}^{\left|T_{s}^{(m)}\right|} z_{s, i}^{(m)}=r_{s}^{(m)}, \quad \forall s \in S^{(m)}, \forall m \in M \\
z_{s, i}^{(m)} \geq 0, \quad \forall i=1, \cdots,\left|T_{s}^{(m)}\right|, \forall s \in S^{(m)}, \forall m \in M .
\end{array}
$$

Let $T=\bigcup_{m \in M}^{s \in S^{(m)}} T_{s}^{(m)}$ be the collection of all multicast trees rooted at any source $s$ for any session $m$. Let $z$ be the vector $\left(z_{s, i}^{(m)}\right)$ where $s \in S^{(m)}, m \in M, i=1, \ldots,\left|T_{s}^{(m)}\right|$, with an arbitrary indexing order for the sources. In problem (38)-(40), let the feasible set defined by (39) and (40) be denoted by $\mathcal{Z}$.

Let $\hat{E}=\bigcup_{m \in M} \hat{E}^{(m)}$ be the collection of all overlay links in all sessions. Let $\hat{H}$ denote the $|\hat{E}| \times|T|$ overlay link-tree incidence matrix associated with the trees in $T$ (i.e. $[\hat{H}]_{\hat{e} t}=1$ if overlay link $\hat{e}$ lies on tree $t$; and $[\hat{H}]_{\hat{e} t}=0$ otherwise). Recall $E$ is the set of underlay links, let $H$ denote the $|E| \times|\hat{E}|$ underlay link-overlay link incidence matrix associated with the overlay links in $\hat{E}$ (i.e. $[H]_{e \hat{e}}=1$ if underlay link $e$ lies on overlay link (underlay path) $\hat{e}$; and $[H]_{e \hat{e}}=0$ otherwise).

For each underlay link $e \in E$, recall that $x_{e}$ is the aggregate flow rate it carries. Let $x$ be the vector $\left(x_{e}\right)_{e \in E}$. It is easy to see $x=H \hat{H} z$. Note that an underlay link $e$ might carry multiple copies of the same file chunk distributed by one tree $t$. Define $\hat{f}_{e}\left(x_{e}\right)=\left(x_{e} / c_{e}+\kappa\right)^{q}$ and $\hat{f}(x)=\sum_{e \in E} \hat{f}_{e}\left(x_{e}\right)$. The objective function, denoted by $f(z)$, is given by

$$
f(z)=\hat{f}(H \hat{H} z)=\sum_{e \in E} \hat{f}_{e}\left(x_{e}\right)=\sum_{e \in E}\left(\frac{x_{e}}{c_{e}}+\kappa\right)^{q},
$$

and (38) can be written as

$$
\begin{array}{cc}
\min & f(z)=\hat{f}(H \hat{H} z) \\
\text { s.t. } & z \in \mathcal{Z} .
\end{array}
$$

The derivative of the objective function $f(z)$ with respect to $z_{s, i}^{(m)}$ is given by

$$
\begin{aligned}
& \frac{\partial f(z)}{\partial z_{s, i}^{(m)}}=\sum_{\hat{e} \in t_{s, i}^{(m)}} \sum_{e \in \hat{e}} \frac{\partial \hat{f}\left(x_{e}\right)}{\partial x_{e}} \\
& =\sum_{\hat{e} \in t_{s, i}^{(m)}} \sum_{e \in \hat{e}} \frac{q}{c_{e}}\left(\frac{x_{e}}{c_{e}}+\kappa\right)^{q-1} .
\end{aligned}
$$

For each $s \in S^{(m)}$ in session $m$, let $i_{s}^{(m)}$ be the index of a minimum-cost tree rooted at $s$ (i.e., with $s$ as the source). That is,

$$
i_{s}^{(m)}(z)=\operatorname{argmin}_{\left\{i: t_{s, i}^{(m)} \in T_{s}^{(m)}\right\}}\left\{\frac{\partial f(z)}{\partial z_{s, i}^{(m)}}\right\} .
$$

Let $i_{s}^{(m)}(k)$ be a short hand of $i_{s}^{(m)}(z(k))$.

\section{Diagonally Scaled Gradient Projection Algorithm}

$$
\begin{gathered}
z(k+1)=\alpha(k) \bar{z}(k)+(1-\alpha(k)) z(k) \\
\bar{z}_{s, i}^{(m)}(k)= \\
\left\{\begin{array}{c}
{\left[z_{s, i}^{(m)}(k)-\delta_{s}^{(m)}(k) \cdot\left(d_{s, i}^{(m)}(k)\right)^{-1}\left(\frac{\partial f(z(k))}{\partial z_{s, i}^{(m)}}-\frac{\partial f(z(k))}{\partial z_{s, i_{s}^{(m)}(k)}^{(m)}}\right)\right]_{+},} \\
\text {if } i \neq i_{s}^{(m)}(k) ; \\
r_{s}^{(m)}-\sum_{1 \leq j \leq\left|T_{s}^{(m)}\right|, j \neq i_{s}^{(m)}(k)} \bar{z}_{s, j}^{(m)}(k), \quad \text { if } i=i_{s}^{(m)}(k),
\end{array}\right.
\end{gathered}
$$

with

$$
d_{s, i}^{(m)}(k)=\sum_{\hat{e} \in t_{s, i}^{(m)} \cup t_{s, i_{s}^{(m)}(k)}^{(m)} \backslash t_{s, i}^{(m)} \cap t_{s, i_{s}^{(m)}(k)}^{(m)}} \sum_{e \in \hat{e}} \frac{q(q-1)}{c_{e}^{2}}\left(\frac{x_{e}(k)}{c_{e}}+\kappa\right)^{q-2} .
$$

The resulting algorithm is still fully distributed.

\section{Convergence Results}

We will show the convergence results of the synchronous gradient projection algorithm under constant step size, i.e., $\delta(k)=\delta$ for all $k$. We will adapt the results from [24] to find an upper bound on the step size $\delta$ that guarantees the global convergence of the synchronous gradient projection algorithm to an optimal solution. Furthermore, with the strictly positive regularization vector $\vec{\kappa}$ (i.e., $\kappa>0$ ), the convergence speed is linear (i.e., geometric). The same convergence results can be said for the case of optimally allocated bandwidth.

In the optimization problem (21), we assume $q \geq 2$, so that $\hat{f}_{e}\left(x_{e}\right)$ is continuous on the interval $[0, \infty)$, tends to $\infty$ as $x_{e}$ approaches $\infty$, and its derivative and second derivative are continuous and positive on $(0, \infty)$. Assuming the links are indexed from 1 to $|\hat{E}|$, the Hessian $\nabla^{2} \hat{f}=\operatorname{diag}\left[\frac{\partial^{2} \hat{f}}{\partial x_{1}^{2}}, \cdots, \frac{\partial^{2} \hat{f}}{\partial x_{|\hat{E}|}^{2}}\right]$ is an $|\hat{E}| \times|\hat{E}|$ diagonal matrix with nonnegative diagonal entries. Furthermore, if $\kappa>0$, the diagonal entries of $\nabla^{2} \hat{f}$ are positive and bounded below by $\min _{e \in \hat{E}}\left\{\frac{q(q-1)}{c_{e}^{2}} \kappa^{q-2}\right\}$.

We assume there is at least one feasible solution, i.e., $z(0) \in$ $\mathcal{Z}$ satisfying $H z(0) \in \prod_{e \in \hat{E}}[0, \infty)$, and define a compact set $\mathcal{Z}_{0}=\{z \in \mathcal{Z} \mid f(z) \leq f(z(0))\}$. Since this set is compact, $f$ must attain a minimum on this set. Hence, there is a $z^{*} \in \mathcal{Z}_{0}$ satisfying $f\left(z^{*}\right)=f^{*}$, where $f^{*}=\min _{z \in \mathcal{Z}_{0}} f(z)$. We call any such $z^{*}$ an optimal solution, and we denote by $\mathcal{Z}^{*}$ the set of optimal solutions. (There may be more than one optimal solutions since, although $\hat{f}$ is strictly convex, $f$ is not.) That is

$$
\mathcal{Z}^{*}=\left\{z \in \mathcal{Z}_{0} \mid f(z)=\min _{z \in \mathcal{Z}_{0}} f(z)\right\} .
$$

For simplicity, we assume the scaling factor $d_{s, i}(k)=1.0$. The convergence results still hold for other scaling factors as long as $\left\{d_{s, i}(k)\right\}$ are bounded between two fixed positive scalars [24] [18].

Let $\delta_{1}=\underline{a} /\left(L \max _{s \in S}\left|T_{s}\right|\right)$, where $L>0$ is an upper bound of the norm of $\nabla^{2} f$ over $\mathcal{Z}_{0}$. 
Lemma 1: For $0<\delta \leq \delta_{1}$, we have for all $k$ that $z(k) \in$ $\mathcal{Z}_{0}$

$f(z(k+1))-f(z(k)) \leq-\left(\frac{\underline{a}}{\delta \max _{s \in S}\left|T_{s}\right|}-\frac{L}{2}\right)\|z(k)-\bar{z}(k)\|^{2}$.

The proof Lemma 1 follows the proof for a similar lemma in [24]. The only change involves substitution of appropriate constants.

Therorem 2: (Globally Convergence) Suppose $\kappa \geq 0$. For any $\delta, 0<\delta<\delta_{1}$, every limit point of $\{z(k)\}$ generated by the synchronous gradient projection algorithm (35)-(37) with $z(0) \in \mathcal{Z}_{0}$ is optimal.

Proof: With the constant step size $\delta<\frac{\underline{a}}{L \max _{s \in S}\left|T_{s}\right|}$, the right-hand side of the inequality (48) is non-positive. Hence, if $\{z(k)\}$ has a limit point, the left-hand side tends to 0 . The algorithm (35)-(37) can be denoted as a function $A(z)$, i.e., $z(k+1)=A(z(k))$. Therefore, $\|z(k)-\bar{z}(k)\| \rightarrow 0$, which implies that for every limit point $\tilde{z}$ of $\{z(k)\}$ we have $\tilde{z}=$ $A(\tilde{z})$. It is easy to show if $\tilde{z}=A(\tilde{z})$, for any $s \in S$, we have $\tilde{z}_{i}^{s}>0$ only if $\frac{\partial f(\tilde{z})}{\partial \tilde{z}_{s, i}} \leq \frac{\partial f(\tilde{z})}{\partial \tilde{z}_{s, j}}, \forall t_{s, j} \in T_{s}$, which is exactly the optimality condition in (25). So $\tilde{z}$ is stationary (Proposition 2.3.2 and Example 2.1.2 in [18]).

When the regularization vector $\vec{\kappa}$ is strictly positive, the diagonal entries of $\nabla^{2} \hat{f}$ are positive and bounded below by $\min _{e \in \hat{E}}\left\{\frac{q(q-1)}{c_{e}^{2}} \kappa^{q-2}\right\}>0$. When $q=2$, the diagonal entries of $\nabla^{2} \hat{f}$ are positive and bounded below by $\min _{e \in \hat{E}}\left\{\frac{q(q-1)}{c_{e}^{2}}\right\}>$ 0 for all $\kappa \geq 0$. In these two cases, all conditions for global geometric convergence required by [24] are satisfied. We can state the global geometric convergence rate for algorithm (35)(37).

Therorem 3: (Globally Geometric Convergence Rate)

Suppose $\kappa>0$. Let $\delta$ satisfy $0<\delta \leq \delta_{1}$. The sequence $\{z(k)\}$ generated by the synchronous gradient projection algorithm (35)-(37) converges to an element of $\mathcal{Z}^{*}$ with an initial feasible $z(0)$ and the convergence rate is linear (i.e., geometric) in the sense that for all $k$,

$$
f(z(k+1))-f^{*} \leq\left(1-D_{5} \delta\right)\left(f(z(k))-f^{*}\right) .
$$

Furthermore, when $q=2$, the above conclusion holds for all $\kappa \geq 0$.

The constants and parameters are as follows. $D_{5}=$ $\underline{a} /\left(D_{4}+\delta_{1}\right), \quad D_{4}=\left((5 L+1)\left(D_{3}\right)^{2}+1+2 \delta_{1}+\right.$ $\left.6 L\left(\delta_{1}\right)^{2} / \underline{a}\right) \max _{s \in S}\left|T_{s}\right|$ and $D_{3}=D \max \left\{1, \delta_{1}\right\}$ for some $D>0$. Moreover, $D$ is bounded above by $D_{1}\left(D_{1}+\left(\sqrt{\max _{s \in S}\left|T_{s}\right|}+1\right) \hat{L}\left\|H^{T}\right\|\right) / \hat{\sigma}$, where $D_{1}=$ $\max \left\{\left\|Q^{-1}\right\| \mid Q\right.$ an invertible submatrix of $\left.H\right\} . \hat{\sigma} \leq \hat{L}$ are any two positive scalars such that the diagonal entries of $\nabla^{2} \hat{f}(H z)$ lie inside $[\hat{\sigma}, \hat{L}]$ for all $z \in \mathcal{Z}_{0}$, where $\nabla^{2} \hat{f}(H z)$ is a positive diagonal matrix.

\section{PRACTICAl Considerations}

The problem formulations in the previous sections omit some details that are practically required. The purpose of this omission is for ease of presentation. These simplified formulations contain the technical core, or the most difficult aspect, of the problem. For the most part, the formulations are without loss of generality. Practical details can be easily incorporated into the formulations. We now address several of these.

\section{A. Overlapping Content}

When some sources share overlapping chunks, we can create a virtual source node that connects all those sources and move all the overlapping chunks to the virtual source. The virtual source has one outgoing virtual link with infinite capacity connecting to each original source. We then arrive at an expanded network. Of course, in actual operation, one of the original physical sources will "act" as the virtual source and run the algorithms assigned to the virtual source.

\section{B. Mixed Architecture of Fixed and Allocated Bandwidth}

In Section II-B and II-C, we see two content distribution scenarios with either fixed or optimally-allocated overlay bandwidth. The latter should achieve better downloading time than the former. However, the latter requires the deployment of our algorithms to all network element, i.e. routers, which is almost certainly impossible. There is an alternative framework in which some routers or devices attached to the routers are deployed with our algorithm, while others not. For instance, our algorithm can be deployed at the cross-ISP links and access links, where the bandwidth is more likely to be small. In this framework, for those physically directly connected router pairs deployed with our algorithms, we model the physical links between them exactly; while for those not directly connected devices, routers and end-systems, we let TCP allocate the end-to-end bandwidth between them and model these end-to-end paths as overlay links. Thus, in our graph of the network, some links are real physical links and others are overlay links with TCP-allocated bandwidth. The algorithm applies as usual.

\section{Network Dynamics and Churn}

Thus far, we assume that the network is stable and no members depart or join until all existing members finish downloading. Since we are considering the distribution of massive files or large collections of files, the assumption is reasonable for the most part. However, we do need to deal with low-degree member churn and network dynamics such as link capacity variations and failures.

If any source owning unique chunks leaves before it finishes disseminating them, those chunks are no longer available in the network. To minimize such risk, in the optimization formulation, we can adjust the requested sending rates $r_{s}$ of the sources. If any source is expected to leave the network soon, it may request (or be assigned) a higher sending rate so that it can spread its chunks to network more quickly.

Other types of network and member dynamics include link failures, the change of link capacities, the arrival of new sources, and the departure and arrival of receivers. As argued in [25], a distributed algorithm has built-in ability to adapt to variations. A distributed algorithm can react rapidly to a local disturbance at the point of disturbance with slower fine tuning in the rest of the network. Such adaptive ability is intimately 
connected with the algorithm's speed of convergence in the static case. Since our algorithm is the result of conscious effort to improve the convergence speed (by diagonal scaling of the gradient algorithm), we believe it is superior in coping with network and member dynamics compared to other similar distributed algorithms. In addition, our distributed algorithm is naturally robust because of the lack of reliance on a central node that might fail. In Section V, we will show a small example of how our algorithm successfully adapts to the departure and arrival of receivers.

\section{Scalability and Hierarchical Partition of Sessions}

In each overlay network, the sources know the complete information of all overlay links and need to run the expensive centralized MDSP algorithm (There is a distributed MDSP algorithm [23]. But the price to pay is the potentially slower speed due to the coordination overhead of distributed operation.). Thus, our gradient algorithm can only deal with distribution sessions with limited size, say several thousands of members in each session. In order to improve the scalability of our algorithm, we shall partition each session and run the algorithm hierarchically, as most scalable network algorithms would do. Though currently we don't have a well-defined way to partition the session, we will show one naive approach to partition a large session in Section V.

\section{E. Asynchronous Algorithm}

Time synchrony is usually difficult to maintain in a large network. An asynchronous version of the scaled gradient algorithm (35)-(37) (or (45)-(47), respectively) could be developed and the corresponding convergence result could be stated following the approach in [26] [24].

\section{Performance Evaluation}

In this section we will compare the performance of our gradient algorithm (GP) with known theoretical bounds, BitTorrent (BT) and Adaptive FastReplica (AFR) [3]. We select BitTorrent because its techniques are interesting and it is the most popular P2P application. We select AFR because it can be thought as using multiple multicast trees for distribution. But only a subset of the trees are allowed, which we call two-level two-phase trees. In each of these trees, the source is connected to one receiver at level 1 , and then the level 1 receiver is connected to all other receivers at level 2. It might appear that such a collection of trees is quite enough for achieving near optimal performance. In an access-constrained network, this is indeed true. However, we will show this is not the case for networks with interior bottlenecks. In that case, a different, maybe larger, collection of trees is needed.

Although we are more interested in network interior bottlenecks, our algorithm can equally deal with bottlenecks at the access links, at the ISP backbone or at the cross-ISP links. Hence, we will consider all these cases. The commercial ISP backbone and cross-ISP topologies are obtained from the Rocketfuel project [27]. In the terminology of BitTorrent, a seed is a source, and a leecher is a receiver. In the previous sections, our objective function is the worst network utilization $\|\vec{\mu}\|_{\infty}$. In the evaluation part, we will focus on the source throughput $R_{s}=r_{s} /\|\vec{\mu}\|_{\infty}$, where $r_{s}$ is the scaled sending rate, and the downloading time $t=L_{s} / R_{s}$, since these are what BitTorrent experiments yield directly. However, recall that the two measures are the two sides of the same coin.

\section{A. The Performance Evaluation Metrics}

1) BitTorrent Simulation: We use the Bittorrent simulator developed by Bharambe et. al. [28]. Since the original simulator only supports access link constraint, we modified the simulator so that it supports general physical network topologies. The overlay link bandwidth, which is the per-connection bandwidth at the underlay, is determined by the max-min bandwidth allocation [17]. In the BitTorrent simulation, we use the following simulation environment.

- Each peer opens 5 uploading connections and one of them is selected by optimistic unchoking, which means that it connects to a random neighbor.

- The seed uses the smart seed policy, which is introduced in [28]. Seeds are with distinct files.

- All the peers join the network at time 0 and continue to be in the network until all of the leechers complete the download.

- All the other parameters follow the regular BitTorrent environment.

Table I summarizes the simulation environment for our test cases.

TABLE I

BITTORRENT SimUlation PARAMETERS

\begin{tabular}{|c||c||c||c|}
\hline & \#Seeds & File Size per Seed & Neighborhood Size \\
\hline Profiles 1-3 & 1 & $62.765 \mathrm{MB}$ & $38-80$ \\
\hline Profile 4 & 1 & $128 \mathrm{MB}$ & $18-40$ \\
\hline Profile 5 & 2 & $64 \mathrm{MB}$ & $18-40$ \\
\hline Profile 6 & 1 & $128 \mathrm{MB}$ & $38-80$ \\
\hline Profile 7 & 1 & $32 \mathrm{MB}$ & $38-80$ \\
\hline
\end{tabular}

2) Adaptive FastReplica: AFR supports single source. To compare with AFR, we partition the physical network into several overlay networks, each for one source according to max-min fairness allocation. AFR constructs two-phase trees as described earlier. From [3], the theoretical throughput of AFR in its best behavior can be computed as

$$
\sum_{i=1, \cdots, m} \min \left\{c_{n_{0} n_{i}}, \min _{j=1, \cdots, m, j \neq i}\left\{c_{n_{i} n_{j}}\right\}\right\},
$$

where $n_{0}$ is the source, $n_{i}, i=1, \cdots, m$ are the receivers, and $c_{n_{i} n_{j}}$ is the end-to-end path (overlay link) capacity between $n_{i}$ and $n_{j}$.

3) Theoretical Bounds: Some theoretical results are used as performance benchmark in our performance comparison. In several studies [29]-[31], researchers have analyzed a model of P2P file sharing among residential users in low accessspeed environment. Each participating end-system has an uplink (to the network) and a downlink with limited capacity. The capacity of the network is considered unlimited. The 
source is to distribute a file to $L$ receivers. Let the uplink (downlink) bandwidth of receiver $i$ be $u_{i}$ ( $d_{i}$, respectively), for $i=1, \cdots, L$. Let the uplink capacity of the source be $u_{s}$. Then, the maximum distribution speed is shown to be

$$
\min \left(u_{s}, \min _{1 \leq i \leq L} d_{i}, \frac{u_{s}+\sum_{1 \leq i \leq L} u_{i}}{L}\right) .
$$

In (51), the three terms are the optimal speeds when the bottleneck is at the source upload link, at a download link, or due to the aggregate upload bandwidth, respectively.

By two-phase distribution, we mean each distribution tree has a depth at most 2. The following fact is known to be true.

Fact 4: In a network with only access-speed constraint, the two-phase distribution [29] achieves the (overlay-network) routing capacity [29], which is (51).

In the single source case, there is a maximum flow from the source to each receiver. The minimum of all these maximum flows will be called the max-flow limit (MFL). The max-flow limit is a throughput (total distribution rate) upper bound. If all nodes but the source are receivers, the max-flow limit is achievable. This result is known as Edmond's Theorem [32] [33].

\section{B. Bottleneck at the Access Links (Profiles 1 to 4)}

In this case, we assume the network has infinite capacity but the access links have finite capacities. We also assume that all access links are deployed with our gradient algorithm. In the four test cases (profile 1-4), we have a single source with uploading bandwidth $u_{s}$. Let $u_{i}$ and $d_{i}$ be leecher (receiver) $i$ 's upload and download bandwidth, respectively.

- Profile 1: $u_{i}=d_{i}=360 \mathrm{Kbps}$ for all 299 receivers, $u_{s}=640 \mathrm{Kbps}$. The download link is the bottleneck.

- Profile 2: $u_{i}=d_{i}=360 \mathrm{Kbps}$ for all 299 receivers, $u_{s}=280 \mathrm{Kbps}$. The source upload link is the bottleneck.

- Profile 3: $d_{i}=360 \mathrm{Kbps}, u_{i}=200 \mathrm{Kbps}$ for all 299 homogeneous receivers, $u_{s}=640 \mathrm{Kbps}$. The aggregate upload bandwidth is the bottleneck.

- Profile $4: d_{i}=360 \mathrm{Kbps}$ for all 100 receivers, $u_{i}=$ $100 \mathrm{Kbps}$ for half of receivers, and $u_{i}=1 \mathrm{Kbps}$ for the rest receivers. $u_{s}=100 \mathrm{Kbps}$. The aggregate upload bandwidth is the bottleneck.

TABLE II

COMPARISON OF DOWNLOADING TIME (MINUTES) AND NUMBER OF ACTIVE TREES.

\begin{tabular}{|c||c||c||c||c|}
\hline & Profile 1 & Profile 2 & Profile 3 & Profile 4 \\
\hline Optimum & 23.8 & 30.6 & 42.4 & 331.4 \\
\hline BT(50\%) & 27.6 & 41.0 & 44.9 & 264.8 \\
\hline BT(95\%) & 28.5 & 41.3 & 49.7 & 428.6 \\
\hline BT(100\%) & 30.4 & 41.5 & 51.0 & 441.8 \\
\hline AFR & 23.8 & 30.6 & 42.7 & 337.9 \\
\hline GP & 23.9 & 30.6 & 43.5 & 333.1 \\
\hline GP \#trees & 3 & 2 & 3 & 53 \\
\hline AFR \#trees & 299 & 299 & 299 & 100 \\
\hline
\end{tabular}

In Table II, the optimal downloading time is computed from by (51). The results indicate that the gradient algorithm obtains near the theoretically optimal solution.
1) Comparison with BitTorrent: Table II shows the time when $50 \%, 95 \%$ and $100 \%$ receivers finish downloading in BitTorrent, respectively. BitTorrent's performance is not bad compared with the optimal value. This was explained in [34], which models the downloading time of BiTtorrent. It shows that, in the case that a flash crowd arrives at the same time, the bandwidth constraint is at the access links, and the receivers stay after they finish downloading, BitTorrent achieves near optimal distribution speed. In Fig. 3 and 4, we show the performance comparison of different distribution schemes under profile 1 and 4 . The results under profile 2 and 3 are omitted for brevity. The download percentage refers to the total amount of data downloaded at each time instance normalized against the total data downloaded at the end of the distribution. Since the two lines have different slopes, we can extrapolate the lines and expect the gradient algorithm to do much better if the file size becomes larger. This observation seems to contradict the conclusion in [34].

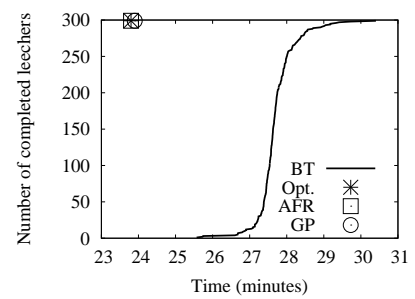

(a)

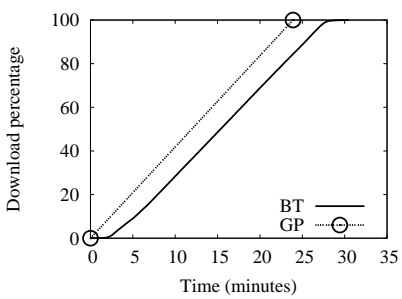

(b)
Fig. 3. Profile 1: (a) number of leechers that have completed download over time; (b) download percentage over time.

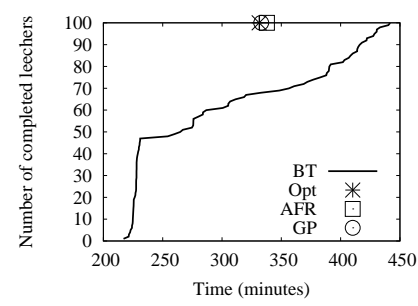

(a)

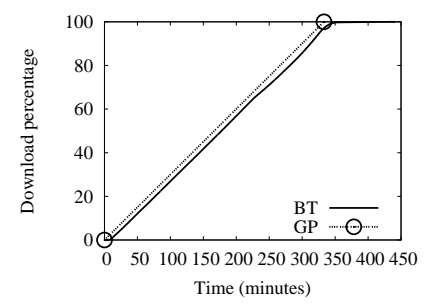

(b)
Fig. 4. Profile 4: (a) number of leechers that have completed download over time; (b) download percentage over time.

2) Comparison with AFR: AFR downloading time is given by (50). Table II shows that AFR's two-phase approach achieves the optimal downloading time when the bottleneck is either at the download link or at the source. But when the bottleneck is due to the aggregate upload bandwidth, AFR fails to achieve the optimum, although we know that some other two-phase solution is optimal. The reason is that, in AFR, every receiver is required to relay all chunks it receives from the source to other receivers. This unnecessary constraint leads to a sub-optimal solution. AFT doesn't allow the breadth-first search tree, but an optimal two-phase tree does. Nevertheless, AFR achieves good performance in this kind of access-limited situation.

We also compare the number of active trees AFR and the gradient algorithm eventually use. The gradient algorithm uses 


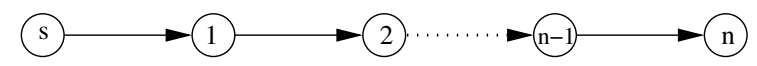

Tree 1: Depth First Search Tree (DFS)

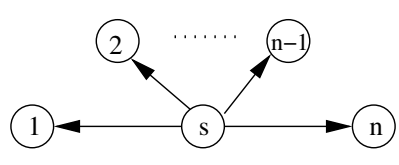

Tree 2: Breadth First Search Tree (BFS)

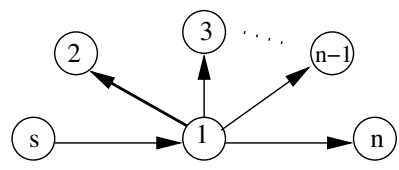

Tree 3: Two-phase Tree
Fig. 5. Structures of trees on the overlay network

fewer trees than AFR. We inspected the active trees. With the access-link constraint, the gradient algorithm uses three kinds of trees, the depth-first search tree (DFS), the breadthfirst search tree (BFS), and a two-phase tree. Fig. 5 shows the structures of the three types of trees on the overlay network. In general, there exists an optimal solution that uses only twophase trees for networks with such a star topology. But it seems that the gradient algorithm prefers the chain-like DFS tree and the fat BFS tree. It may appear counter-intuitive that such a chain-like distribution path is preferred because the chain seems to involve largest delay. However, this is in fact not true because of our fluid model of traffic and because we do not consider propagation delay. The bit that arrives at node 1 from the source can immediately be transmitted to node 2, and to node 3 , so on. We leave it to future work on how to incorporate the propagation delay in optimal tree selection. Table III shows that, when the bottleneck is at either the download links or the source, the gradient algorithm naturally prefers the DFS tree. When the aggregate upload bandwidth is the bottleneck, we have to distribute the chunks over the twophase trees. In addition, the more heterogenous the receivers are, the more two-phase trees we need and the more bandwidth is allocated to the two-phase trees. The key conclusion here is that the gradient algorithm is able to find the best distribution trees for the particular network environment. Without the help of the gradient algorithm, what types of trees are selected is not always obvious.

TABLE III

THE Distribution OF BANDWIDTH ALlOCATED FOR DIFFERENT TREES

\begin{tabular}{|c||c||c||c||c|}
\hline & Profile 1 & Profile 2 & Profile 3 & Profile 4 \\
\hline DFS & $99.4 \%$ & $99.999 \%$ & $98.91 \%$ & $1.93 \%$ \\
\hline BFS & $0.26 \%$ & 0 & $0.754 \%$ & $0.95 \%$ \\
\hline Two-phase & $0.33 \%$ & $0.001 \%$ & $0.333 \%$ & $97.1 \%$ \\
\hline
\end{tabular}

\section{Bottleneck at the Internal of ISP Backbone (Profile 5)}

In this case, we assume all access links have unlimited bandwidth, and congestion happens at the core network. We wish to see how our algorithm performs in infrastructuremode content distribution. We did experiments with the ISP Sprintlink's backbone obtained from [27]. The network has 315 backbone nodes and 1944 links. It is the largest backbone ISP with the highest node degree among the six commercial backbone networks that the RocketFuel project provides. We attach 100 peers with unlimited access bandwidth randomly to some backbone nodes, with at most one peer per backbone node. One may think a peer is a large content distribution server cluster. Among the 100 peers, we choose 2 sources with the normalized sending rates $r_{s}=1.0$ (dimensionless value).

We did several experiments with the link capacities uniformly distributed in some range. The actual link capacity data is unavailable. We find the gradient algorithm often gives trivial optimal solutions. After inspecting the solutions and the network graphs, it turns out that the ISP backbone is poorly connected. There are many links that lies on all the routing paths between one peer and all other peers, which means if any one of these critical links is removed, at least one peer will be disconnected. If these critical links do not have much larger capacity than other links, they are likely to become the bottleneck. The gradient algorithm is able to locate the bottleneck immediately. Other five ISP backbones show the same property. Presumably in reality, the ISPs are aware of such links and would ensure they have very large bandwidth so that they are never the bottleneck. In order to test our algorithm in this non-trivial scenario, we assign the same bandwidth, 1000 , to all backbone links. Then, we scale up the bandwidth of all critical links (those links that, when removed, will leave some peers disconnected in the overlay) to be large enough so that they are not the bottleneck.

We did three tests on Profile 5.

- (Test a) Our algorithm is deployed at all links. This is the case of optimally-allocated overlay bandwidth.

- (Test b) Our algorithm is deployed only at the peers. The overlay bandwidth between each pair of peers is fixed by the max-min allocation. The 100 peers form a single overlay network.

- (Test c) Our algorithm is deployed only at the peers. In order to compare with AFR, we partition the backbone into two overlay networks, one for each source. Note that the max-flow limit is achievable in this case.

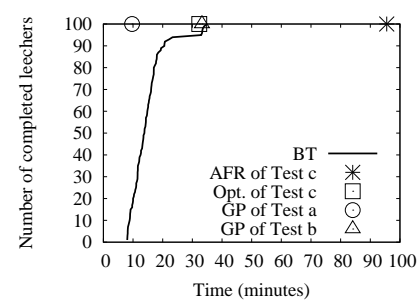

(a)

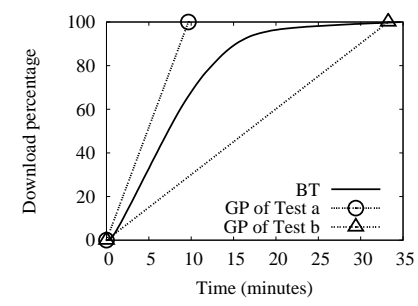

(b)
Fig. 6. Profile 5. (a) Number of receivers that have completed download over time; (b) download percentage over time.

1) Comparison with BitTorrent: Fig. 6 shows that, in Test a, the downloading time in the gradient algorithm is only $30 \%$ of that in BitTorrent. BitTorrent is unable to give good performance when the core network is congested. But in Test b, after the overlay bandwidth is fixed, the optimal downloading time is much higher than that of Test a, and almost equal to BitTorrent's time. In the figure, the download percentage refers to the total amount of data downloaded at each time instance 
normalized against the total data downloaded at the end of the distribution. Since the lines have different slopes, we can extrapolate the lines and expect the gradient algorithm to do much better if the file size becomes larger.

2) Comparison with AFR: Fig. 6 also shows that, in Test c, the gradient algorithm approaches the max-flow limit while AFR achieves something far from the optimum. When the congestion happens at the core network, the two-phase trees alone fail to give good solution.

3) Convergence Speed: Fig. 7 shows the convergence of the algorithm in Test $\mathrm{a}, \mathrm{b}$ and $\mathrm{c}$ respectively. The time spent on one iteration is about one round trip time plus the time to compute the MDSP. It seems that the algorithm that optimally allocates the overlay bandwidth converges much faster than the algorithm with fixed overlay bandwidth. This has to do with the fact that, in the final solution, Test a has 92 active trees, while Test b has totally 4746 active trees. It is possible Test $b$ has another optimal or nearly optimal solution that has much fewer trees. Finding solutions with fewer trees should improve convergence speed, and is an important direction to pursue.

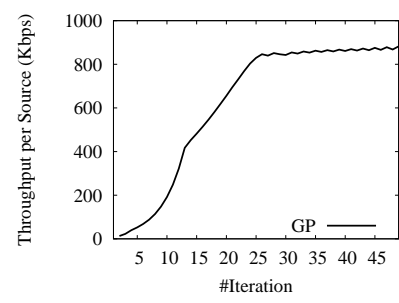

(a)

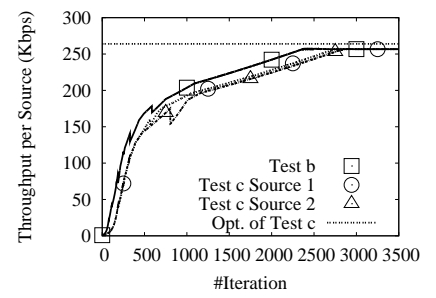

(b)
Fig. 7. Profile 5. Convergence of throughput. Two sources with $r_{s}=1.0$ (a) Test a; (b) Test b and c.

\section{Bottleneck at the Cross-ISP Links (Profile 6-7)}

In reality, the cross-ISP links are often the bandwidth bottleneck. The goal here is to evaluate the effectiveness of our algorithm in handling the bottleneck at cross-ISP links when it is deployed at ISP gateways. We did experiments both on an artificial small cross-ISP network and the crossISP network obtained from the RocketFuel project. We created scenarios where congestion happens at the cross-ISP links. Our algorithm is deployed at all access links and cross-ISP links. Hence, we're able to run the algorithm to optimally allocate the overlay bandwidth.

- Profile 6 (P6): 6 completely connected ISPs with 30 cross-ISP links. Each cross-ISP link has a capacity of 1000. 300 peers are attached to the ISPs, 50 per ISP, with sufficient access bandwidth. A single source is attached to one ISP.

- Profile 7 (P7): (RocketFuel topology): 69 ISPs connected with 1336 links. The cross-ISP link capacities are uniformly distributed on $(100,1000) .500$ peers are randomly attached to the ISPs with sufficient bandwidth. A single source is attached to one ISP.
Note that, in the case of a single source, congestion at the cross-ISP links and each ISP containing some peers, the maxflow limit is achievable.

TABLE IV

DownLOADING TIME (MINUTES) COMPARISON OF PROFILE 6 AND 7

\begin{tabular}{|l||c||c||c||c||c||c|}
\hline & $\mathrm{BT}(50 \%)$ & $\mathrm{BT}(95 \%)$ & $\mathrm{BT}(100 \%)$ & GP & AFR & MFL \\
\hline P6 & 16 & 19.5 & 19.6 & 3.8 & 142.7 & 3.4 \\
\hline P7 & 5.83 & 26.5 & 90 & 8.74 & 131.2 & 8.72 \\
\hline
\end{tabular}

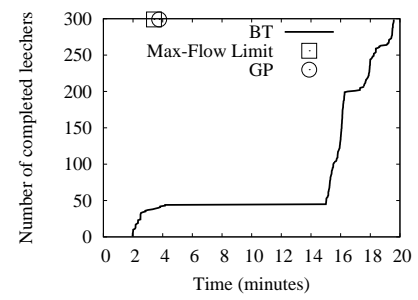

(a)

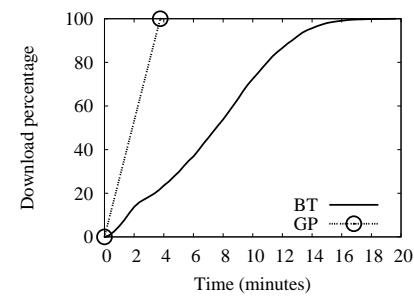

(b)
Fig. 8. Profile 6. (a) Number of receivers that have completed download over time; (b) download percentage over time.

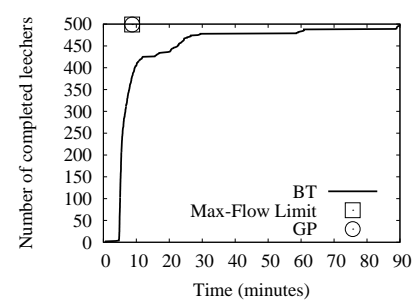

(a)

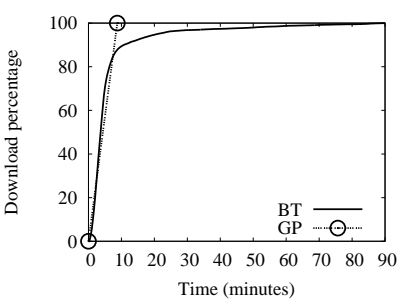

(b)
Fig. 9. Profile 7. (a) Number of receivers that have completed download over time; (b) download percentage over time.

In both profiles 6 and 7, the gradient algorithm approaches the max-flow limit and beats BitTorrent and AFR by a large amount, up to a factor of 10 (Tabel IV: $50 \%, 95 \%$ and $100 \%$ are the percentage of the peers that have finished downloading. Also see Fig. 8 and 9.). We found, with more peers per ISP, BitTorrent's performance deteriorates. FastReplica's performance is far worse than both the gradient algorithm and BitTorrent, and we didn't even show it in Fig. 8 and 9.

Usually, cross-ISP traffic is more expensive. We investigated the traffic redundancy over the cross-ISP links. With neither inside ISP congestion nor access speed constraint, ideally, each destination ISP should receive only one copy of each chunk from other ISPs and the source ISP should not receive any copy from other ISPs. In Profile 6, we inspected the active (optimal) trees the algorithm constructed and found that each destination ISP indeed only received one copy from other ISPs, but the source ISP might receive some copies from other ISPs. Suppose the normalized cross-ISP traffic under the ideal distribution is 1.0. We found the cross-ISP traffic was 1.103 in the gradient algorithm and 5.982 in BitTorrent. But in Profile 7 , we found the destination ISPs received multiple copies from other ISPs in the gradient algorithm. This is because, in Profile 
6, each max-flow between the source ISP and the destination ISP has the same value. This is not the case in Profile 7. Thus, the optimal solution does allow multiple copies to be sent to one destination ISP. Again, if the normalized cross-ISP traffic under the ideal distribution is 1.0 , then the traffic is 2.22 in the gradient algorithm and 9.72 in BitTorrent.

\section{E. Arrival and Departure Dynamics (Profile 8)}

Here, we wish to examine how well the distributed gradient algorithm copes with the peer arrival and departure dynamics. We applied the algorithm with optimally allocated overlay bandwidth on a small star network with receivers arrive and depart randomly. All peers have sufficient download capacities; the receivers each have upload bandwidth $200 \mathrm{Kbps}$; and the source has upload bandwidth $640 \mathrm{Kbps}$. In Phase-I, we have one source and 10 receivers; in Phase-II, 2 receivers leave; in Phase-III, 5 new receivers arrive. Assume at the beginning of Phase-III the source has distributed half of the chunks to the 8 existing receivers. We assume at the end of Phase-III, all 13 receivers finish at the same time (though it's unfair). Thus, we set up two more sessions at PhaseIII: one consists of the 5 new receivers and the original source for downloading the second half of the chunks; and the other consists of the 5 new receivers, the source and the 8 existing receivers (now also serving as sources) for downloading the first half of the chunks. Thus, in Phase-III, we have 3 sessions, and in the 3rd session, we have multiple sources with overlapping chunks. Fig. 10 shows the algorithm adapts to the dynamics quickly.

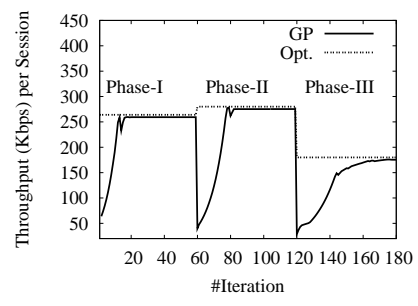

Fig. 10. Dynamic departure and arrival of receivers

\section{AdDitional Related Works}

In our optimization-based approach, we follow the tradition of Kelly et. al. [35] and Low et. al. [36] on optimal flow control/bandwidth allocation. Many recent papers extended this approach and solved networking problems by collective actions taken across networking layers, especially in wireless networks e.g., [37]-[40]. Several other related studies, either in topics or methods, are [41]-[44].

\section{CONCLUSION}

This paper represents a systematic study on how best to conduct content distribution using advanced P2P techniques. In response to growing massive content that threatens to congest the core network, our objective is to manage the network congestion not only at the access links but throughout the network, especially at cross-ISP links. We showed that this objective is "equivalent" to speeding up content distribution. The main contribution of this paper is that we envision optimal content distribution as a multicast tree packing problem, and we derive a distributed algorithm for solving the problem. The tree-packing framework is also useful for contemplating existing P2P swarming/collaborative downloading techniques, by asking the questions: What kind of trees do existing algorithms use? How are the trees selected? And how is bandwidth assigned to the trees? Hence, our framework has the potential to provide a unified understanding of $\mathrm{P} 2 \mathrm{P}$ distribution techniques. Finally, our distributed algorithm is based on a specialized gradient projection algorithm for optimization under simplex constraints and we develop a scaled version of it. Our computation experiences show that it has much faster convergence than the more frequently used subgradient algorithm.

\section{REFERENCES}

[1] BitTorrent Website, http://www.bittorrent.com/.

[2] L. Cherkasova and J. Lee, "Fastreplica: Efficient large file distribution within content delivery networks," in Proceedings of the 4th USITS, Seattle, WA, March 2003.

[3] J. Lee and G. de Veciana, "On application-level load balancing in FastReplica," Computer Communications, vol. 30, no. 17, pp. 32183231, November 2007.

[4] D. Kostić, A. Rodriguez, J. Albrecht, and A. Vahdat, "Bullet: high bandwidth data dissemination using an overlay mesh," in Proceedings of 19th ACM Symposium on Operating Systems Principles (SOSP 'O3), October 2003.

[5] D. Kostić, R. Braud, C. Killian, E. Vandekieft, J. W. Anderson, A. C. Snoeren, and A. Vahdat, "Maintaining high bandwidth under dynamic network conditions," in Proceedings of USENIX Annual Technical Conference, 2005.

[6] B.-G. Chun, P. Wu, H. Weatherspoon, and J. Kubiatowicz, "ChunkCast: An anycast service for large content distribution," in Proceedings of the Internaltional Workshop on Peer-to-Peer Systems (IPTPS), February 2006.

[7] K. Park and V. S. Pai, "Scale and performance in the CoBlitz largefile distribution service," in Proceedings of the 3rd USENIX/ACM Symposium on Networked Systems Design and Implementation (NSDI), San Jose, CA, May 2006.

[8] D. Bickson, D. Malkhi, and D. Rabinowitz, "Efficient large scale content distribution," in Proceedings of the 6th Workshop on Distributed Data and Structures (WDAS'2004), Lausanne, Switzerland, July 2004.

[9] K. Wieland, "DigiWorld 2006: Voice and low prices drive FTTH in Japan," Telecommunications Online, Nov. 15 2006, http://www. telecommagazine.com.

[10] J. George, "FTTH design with the future in mind," Broadband Properties, September 2005

[11] K. Cho, K. Fukuda, H. Esaki, and A. Kato, "The impact and implications of the growth in residential user-to-user traffic," in Proceedings of ACM Sigcomm, Pisa, Italy, September 2006.

[12] S. Q. Zhuang, B. Y. Zhao, A. D. Joseph, R. H. Katz, and J. Kubiatowicz, "Bayeux: An architecture for scalable and fault-tolerant wide-area data dissemination," in Proceedings of the 11th International Workshop on Network and Operating Systems Support for Digital Audio and Video (NOSSDAV) , June 2001.

[13] Akamai Website, http://www.akamai.com.

[14] J. Cannons, R. Dougherty, C. Freiling, and K. Zeger, "Network routing capacity," IEEE Transactions on Information Theory, vol. 52, no. 3, pp. 777-788, March 2006.

[15] M. Grotchel, A. Martin, and R. Weismantel, "Packing Steiner trees: a cutting plane algorithm and computation," Mathematical Programming, vol. 72, pp. 125-145, 1996.

[16] K. Jain, M. Mahdian, and M. R. Salavatipour, "Packing Steiner trees," in Proceedings of the fourteenth annual ACM-SIAM symposium on Discrete algorithms (SODA '03), 2003.

[17] D. Bertsekas and R. Gallager, Data Networks, 2nd ed. Prentice Hall, 1991. 
[18] D. Bertsekas, Nonlinear Programming, 2nd ed. Athena Scientific, 1999.

[19] R. Madan, Z. Luo, and S. Lall, "A distributed algorithm with linear convergence for maximum lifetime routing in wireless networks," in Proceedings of the Allerton Conference on Communication, Control, and Computing, September 2005.

[20] D. Bertsekas and J. N. Tsitsiklis, Parallel and Distributed Computation: Numerical Method. Athena Scientific, 1997.

[21] R. E. Tarjan, "Finding optimum branchings," Networks, Vol. 7, pp. 25$35,1977$.

[22] L. F. P. M. Camerini and F. Maffioli, "A note on finding optimum branchings," Networks, Vol. 9, pp. 309-312, 1979.

[23] P. A. Humblet, "A distributed algorithm for minimum weight directed spanning trees," IEEE Transactions on Communications, pp. 756-762, June 1983.

[24] Z.-Q. Luo and P. Tseng, "On the rate of convergence of a distributed asynchronous routing algorithm," IEEE Transcations on Automatic Control, vol. 39, pp. 1123-1129, May 1994.

[25] R. G. Gallager, "A minimum delay routing algorithm using distributed computation," IEEE Transactions on Communications, pp. 73-85, January 1977.

[26] J. N. Tsitsiklis and D. P. Bertsekas, "Distributed asynchronous optimal routing in data networks," IEEE Transactions On Automatic Control, vol. AC-31, pp. 325-332, 1986.

[27] Rocketfuel: An ISP Topology Mapping Engine, University of Washington, http://www.cs.washington.edu/research/networking/rocketfuel/.

[28] A. R. Bharambe and C. Herley, "Analyzing and improving BitTorrent performance," Microsoft Research, Tech. Rep. MSR-TR-2005-03, 2005.

[29] R. Kumar and K. Ross, "Peer-assisted file distribution: The minimum distribution time," in IEEE Workshop on Hot Topics in Web Systems and Technologies (HOTWEB), 2006.

[30] D. M. Chiu, R. W. Yeung, J. Huang, and B. Fan, "Can network coding help in P2P networks?" in The fourth International Symposium on Modeling and Optimization in Mobile, Ad Hoc and Wireless Networks, 2006.

[31] J. Mundinger, R. R. Weber, and G. Weiss, "Optimal scheduling of peerto-peer file dissemination," arXiv e-print service, June 2006, http://arxiv. org/abs/cs.NI/0606110.

[32] Y. Wu, P. A. Chou, and K. Jain, "A comparison of network coding and tree packing," in The Proceedings of IEEE International Symposium on Information Theory (ISIT), June 2004.

[33] J. Edmonds, "Edge-disjoint branchings," in Combinatorical Algorithms, R. Rustin, Ed. Academic Press, 1973, pp. 91-96.

[34] D. Qiu and R. Srikant, "Modeling and performance analysis of BitTorrent-like peer-to-peer networks," in Sigcomm'04, 2004.

[35] F. Kelly, A. Maulloo, and D. Tan, "Rate control for communication networks: shadow price, proportional fairness and stability," Journal of the Operational Research Society, vol. 49, pp. 237-252, 1998.

[36] S. H. Low and D. E. Lapsley, "Optimization flow control - I: Basic algorithm and convergence," IEEE/ACM Transactions on Networking, vol. 7 , no. 6, pp. 861-874, 1999.

[37] X. Lin and N. B. Shroff, "Joint rate control and scheduling in multihop wireless networks," in Proceedings of the 43rd IEEE CDC, 2004.

[38] A. Eryilmaz and R. Srikant, "Fair resource allocation in wireless networks using queue-length based scheduling and congestion control," in Proceedings of the IEEE Infocom 2005, Miami, USA, March 2005.

[39] J. Wang, L. Li, S. H. Low, and J. C. Doyle, "Cross-layer optimization in TCP/IP networks," IEEE/ACM Transactions on Networking, vol. 13, no. 3, pp. $582-595$, June 2005.

[40] L. Chen, S. H. Low, M. Chiang, and J. C. Doyle, "Cross-layer congestion control, routing and scheduling design in ad hoc wireless networks," in Proceedings of the IEEE Infocom 2006, Barcelona, Spain, April 2006.

[41] Y. N. Wu and S.-Y. Kung, "Distributed utility maximization for network coding based multicasting: a shortest path approach," IEEE Journal on Selected Areas in Communications, vol. 24, no. 8, pp. 1475-1488, Aug. 2006.

[42] P. Key, L. Massouliè, and D. Towsley, "Path selection and multipath congestion control," in Proceedings of INFOCOM 2007, May 2007.

[43] R. Bindal, P. Cao, W. Chan, J. Medval, G. Suwala, T. Bates, and A. Zhang, "Improving traffic locality in BitTorrent via biased neighbor selection," in Proceedings of the International Conference on Distributed Computing Systems (ICDCS'06), 2006.

[44] H. Zhang, G. Neglia, D. Towsley, and G. L. Presti, "On unstructured file sharing networks," in Proceedings of INFOCOM, May 2007.

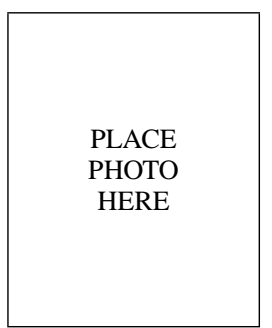

Xiaoying Zheng is a $\mathrm{PhD}$ candidate in the Department of Computer and Information Science and Engineering at the University of Florida. She received her bachelor's and master's degrees in computer science and engineering from Zhejiang University, P.R. China, in 2000 and 2003 respectively. Her research interests include applications of optimization theory in networks, peer-to-peer overlay networks, content distribution and congestion control.

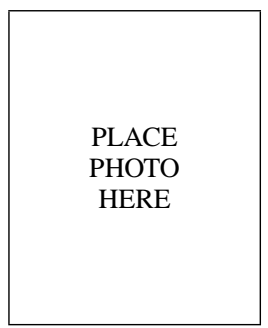

Chunglae Cho received the B.S. and M.S. degrees in computer science from Pusan National University, Korea, in 1994 and 1996, respectively. He worked as a research staff member at Electronics and Telecommunications Research Institute, Korea, between 2000 and 2005. He is currently working toward the Ph.D. degree in the Computer and Information Science and Engineering department at the University of Florida, Gainesville, FL. His research interests are in computer networks, including networks and wireless networks.

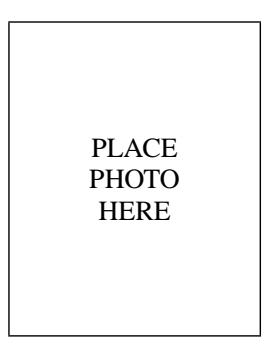

Ye Xia is an assistant professor at the Computer and Information Science and Engineering department at the University of Florida, starting in August 2003. He has a PhD degree from the University of California, Berkeley, in 2003, an MS degree in 1995 from Columbia University, and a BA degree in 1993 from Harvard University, all in Electrical Engineering. Between June 1994 and August 1996, he was a member of the technical staff at Bell Laboratories, Lucent Technologies in New Jersey. His research interests are in computer networking area, including performance evaluation of network protocols and algorithms, congestion control, resource allocation, and load balancing on peer-to-peer networks. He is also interested in probability theory, stochastic processes and queueing theory. 